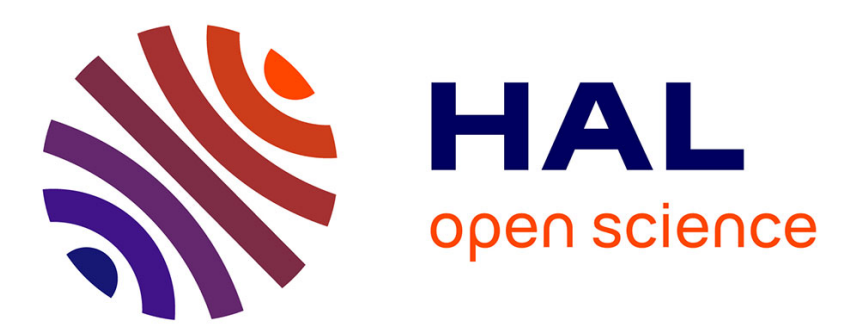

\title{
Analysis of Thin Film Parylene-Metal-Parylene Device Based on Mechanical Tensile Strength Measurement
} Seonho Seok, Hyungdal Park, Wonsuk Choi, Ockchul Kim, Jinseok Kim

\section{To cite this version:}

Seonho Seok, Hyungdal Park, Wonsuk Choi, Ockchul Kim, Jinseok Kim. Analysis of Thin Film Parylene-Metal-Parylene Device Based on Mechanical Tensile Strength Measurement. 2019 Symposium on Design, Test, Integration \& Packaging of MEMS and MOEMS (DTIP), May 2019, Paris, France. hal-02373523

\section{HAL Id: hal-02373523 \\ https://hal.science/hal-02373523}

Submitted on 21 Nov 2019

HAL is a multi-disciplinary open access archive for the deposit and dissemination of scientific research documents, whether they are published or not. The documents may come from teaching and research institutions in France or abroad, or from public or private research centers.
L'archive ouverte pluridisciplinaire HAL, est destinée au dépôt et à la diffusion de documents scientifiques de niveau recherche, publiés ou non, émanant des établissements d'enseignement et de recherche français ou étrangers, des laboratoires publics ou privés. 


\title{
Analysis of Thin Film Parylene-Metal-Parylene Device Based on Mechanical Tensile Strength Measurement
}

\author{
Seonho Seok ${ }^{1}$, HyungDal Park ${ }^{2}$, Wonsuk Choi ${ }^{2}$, Ockchul Kim², and Jinseok Kim² \\ Center for Nanoscience and Nanotechnology (C2N), University-Paris-Saclay, Orsay, France ${ }^{1}$ \\ Center for Bionics, Korea Institute of Science and Technology (KIST), Seongbuk-gu, Seoul, Republic of Korea ${ }^{2}$ \\ Email: seonho.seok@u-psud.fr
}

\begin{abstract}
This paper presents an FEM analysis and experiment of parylene-metal-parylene flexible substrate for implantable medical devices. Tensile strength measurement of the parylene-metal-parylene has been carried out and corresponding FEM modeling and simulation has been done to understand its mechanical behaviour. Besides, frequently encountered metal delamination on parylene substrate has been studied based on cohesive zone model of interface between the two materials.
\end{abstract}

Keywords-FEM (Finite Element Method), Parylene, Neural probe, Implantable

\section{INTRODUCTION}

Many research efforts have been made to develop and improve of the prosthetic hands and arms for the amputees, and in recent years much progress has been observed in the development of life-like robotic hands and the means of controlling them with greater degree of freedom. For this purpose, micro-electro-mechanical systems (MEMS) technlogies have been used to fabricate neural inteface probes [1-3]. However, existing MEMS-based neural electrodes would have limitation on neural interface due to its material characteristics. Thus, flexible neural electrodes have been recently proposed to minimize mechanical mismatch between the electrode and tissue after the electrode's implantation for a stable long-term recording. To this sense, peripheral neural interface (PNI) devices have been appeared to retrieve and send neural signals directly from and to the residual or existing peripheral nerves in this field [4]. Recently, thin film flexible polymeric devices are being used for measuring nerve impulse from the central or peripheral nerve systems. While thin-film polymeric devices are flexible and biocompatible, they are prone to delamination and carry concerns about their mechanical robustness [5]. A nanopillar array created by plasma etching could be used to enhance adhesion among different materials in the parylene-metal-parylene system [6]. ALD (Atomic layer deposition) $\mathrm{Al}_{2} \mathrm{O}_{3}$ combined with the silane adhesion promoter A-174 would increase adhesion force between two parylene films [5]. Parylene material has been shown that mechanical properties can be maintained after stored in PBS (Phosphate Buffered Saline) solution up to 12 months [7]. Therefore, mechanical or chemical treatments of the interface between polymer substrate and metal film is frequently required [8].
In this paper, mechanical characterization of parylene based peripheral neural device has been presented. FEM models and simulation have been used to comprehend its mechanical behaviour and the effect of metal adhesion form on possible metal delamination on parylene substrate. For example, stress distribution of a polymeric neural probe winding a nerve has been presented in Fig. 1. There could be a critical failure near the probe edge due to applied axial force during implantation or operation. Note that the stress distribution on the neural probe depends on the way how it is winded about the nerve. Therefore, it is highly required to characterize mechanical properties of the thin-film probe device.

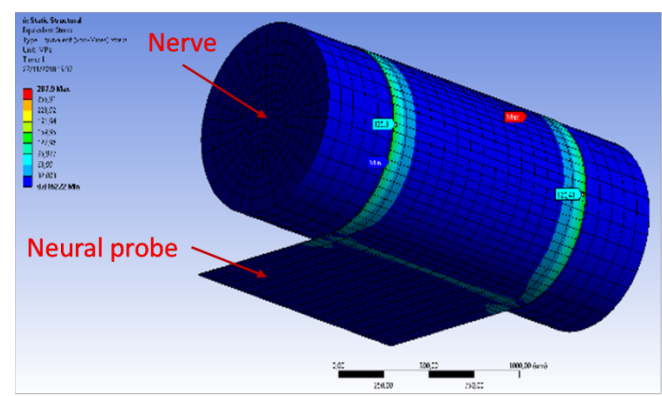

Fig. 1 An example of stress distribution of a neural probe winding a nerve

\section{MECHANICAL CHARACTERIZATION OF PARYLENE-METAL- PARYLENE SUBSTRATE}

Neural probe based on parylene typically has multi-layer structure consisting of top parylene, center metal electrode, and bottom parylene. Thus, a parylene-metal-parylene substrate has been fabricated to extract mechanical parameters required for FEM modeling. Fig. 2 shows the conceptual drawing of the test sample. The metal lines in between the parylene layers would be used as resistance monitor during tensile loading test. 


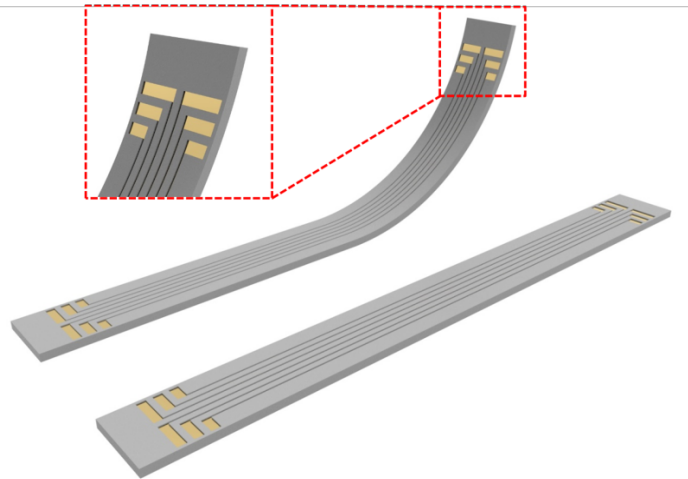

Fig. 2 Conceptual drawing of test sample for parylene-metal-parylene substrate

Fig. 3 shows fabrication process of test parylene sample; (a) Si substrate is prepared. (b) Aluminium metal of $1 \mu \mathrm{m}$ thickness has been evaporated as release layer. (c) Bottom parylene-C layer of $5 \mu \mathrm{m}$ has been deposited on top of the Al layer. (d) Metal line has been patterned with PR/Al mask using ICP RIE. It should be noted that the parylene surface has been etched by CF4 gas to create nanopillar at the parylene surface as shown in Fig. 4. (e) Top parylene-C layer of $5 \mu \mathrm{m}$ has been deoposited. (f) $\mathrm{Al}$ etch mask has been defined with PR mask by ICP RIE and then top parylene layer is pattened by using $\mathrm{O} 2$ plasma etch. (g) Al release layer has been etched and parylene test sample has been released.

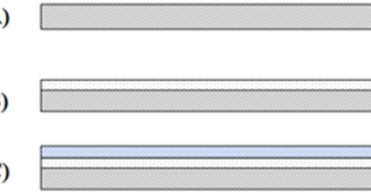

(D)

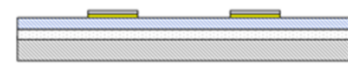

Silicon Aluminum

(E)

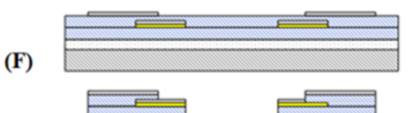

(G)

(H)

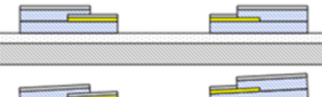
Parylene
Fig. 3 Fabrication process of the test parylene sample

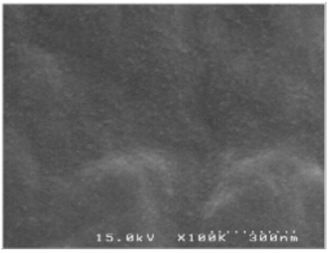

(a) untreated

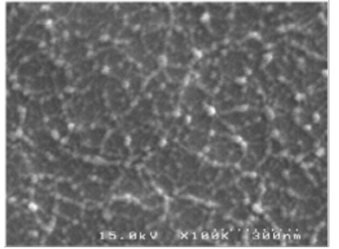

(b) etched by CF4
Fig. 4 Parylene surface modification

After metal electrode patterning, it has been observed that some electrodes have been delaminted from the parylene substrate as shown in Fig. 5. The interface between metal electrode and parylene has the lowest adhesion compared with other interfaces in parylene-metal-parylene system [6]. As maximal adhesion at metal-parylene interface is less than 2.5 $\mathrm{MPa}$ even with CF4 etch, it is included in FEM model of the metal delamination from parylene substrate [6].

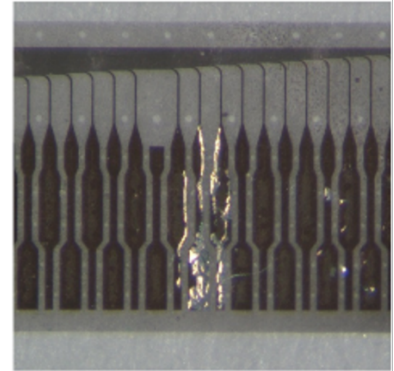

Fig. 5 Metal delamination on parylene substrate

Fig. 6 shows the SEM pictures and the profile measurement of the fabricated parylene-metal-parylene substrate; gold signal line of $330 \mathrm{~nm}$ in thickness is sandwiched by top and bottom parylene layer of $5 \mu \mathrm{m}$ in thickness and thus total thickness of the parylene-metal-parylene is approximately $10 \mu \mathrm{m}$. Note that the top parylene layer is intentionally patterned to measure its thickness and the test structure is the same one as the neural probe.
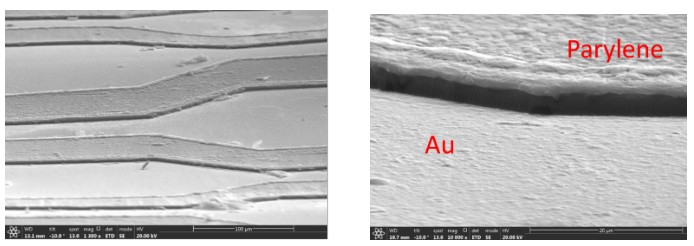

(a) SEM pictures

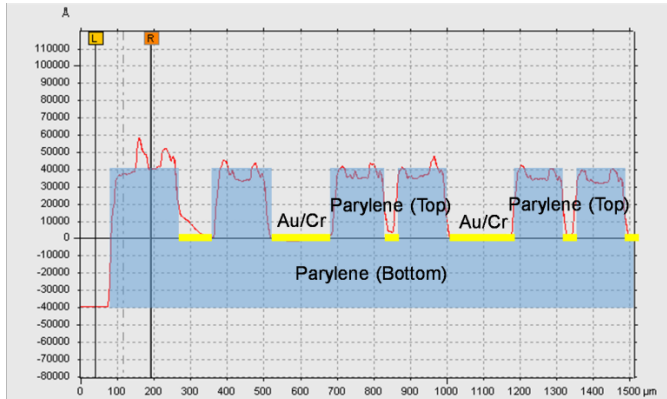

(b) Measure profile

Fig. 6 Structure of the parylene-metal-parylene substrate for mechanical characterization

\section{A. Mechanical characterization}

To find mechanical properties of the parylene material and its mechanical behaviour, a tensile strength test device has been fabricated as shown in Fig. 7. The test device has size of 45 $\mathrm{mm}$ in length, $1.5 \mathrm{~mm}$ in width and $10 \mu \mathrm{m}$ in thickness and two gold lines having $330 \mathrm{~nm}$ in thickness and $200 \mu \mathrm{m}$ in width are placed in between the two paylene layers. The tensile strength test with Shimadzu EZ-S machine has been carried out as shown in Fig. 8. Force is applied to one end of the test device with speed of $1 \mathrm{~mm}$ per minute while the other end is fixed. Elongation length of the parylene test sample is recorded as a function of the applied force and then stressstrain curves are extracted as shown in Fig. 10. The extracted 
mechanical parameters of the test parylene-metal-parylene are Young's modulus of $2.67 \mathrm{GPa}$ and Yield strength of $30.7 \mathrm{MPa}$. Note that the extracted mechanical parameters are averaged value of 3 test samples. Fig. 11 shows the fractured test parylene sample.

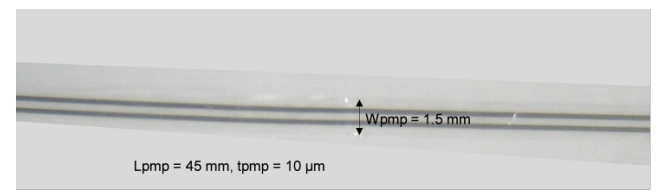

Fig. 7 Test device for tensile strength test

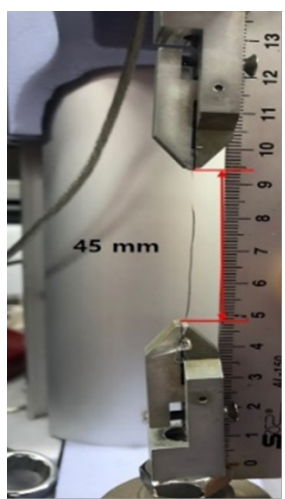

(a) mounting

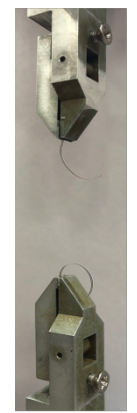

(b) fracture
Fig. 8 Tensile strength test; (a) mounting of test parylene device (b) fracture of test parylene device

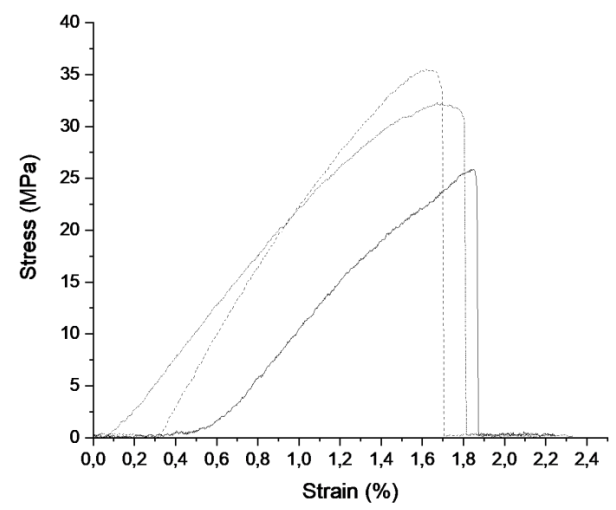

Fig. 9 Tensile test results; Stress-strain curves
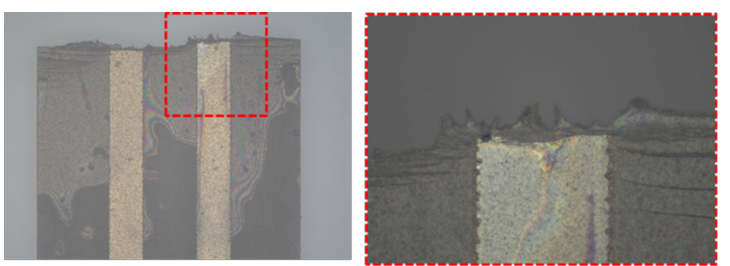

Fig. 10 Fractured test parylene sample from tensile test of parylene-metal-parylene having $10 \mu \mathrm{m}$ in thickness and 5 $\mathrm{mm}$ in length with the same width as the test sample has been simulated to understand mechanical behaviour of the parylene system. Material properties of the model are $3 \mathrm{GPa}$ of Young's modulus and 0.4 of poisson ratio. The value of the poisson ratio is chosen as does the typical polymeric materials. Displacement load of $500 \mu \mathrm{m}$ has been applied into one end, which the other end is fixed. Fig. 11 shows stress distribution of gold electrode and parylene substrate. Maximal stress is found near the fixed end which is corresponding to the test experiment as some cracked parylene near the fractured side of the test sample has been found as shown in Fig. 10. The test parylene sample has fractured at applied strain of $1.7 \%$ as shown in Fig. 12. Strain at break of $1.7 \%$ is too small compared with typical parylene mechanical properties [9]. Simulation result also shows tensile strength of the parylene material is reached when $10 \%$ strain is applied. Note that tensile strength of the parylene-C material is $69 \mathrm{MPa}$ [9].
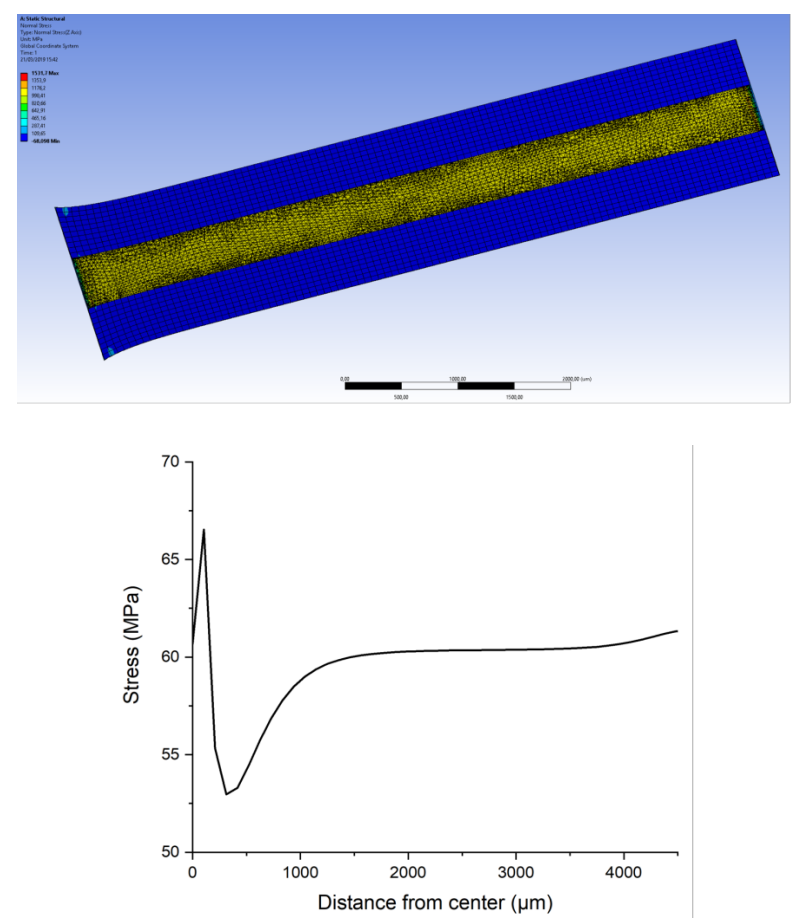

Fig. 11 Stress distribution during tensile strength test

\section{B. FEM modeling}

A finite element model (FEM) has been built to study mechanical behaviour of the parylene device. An FEM model 


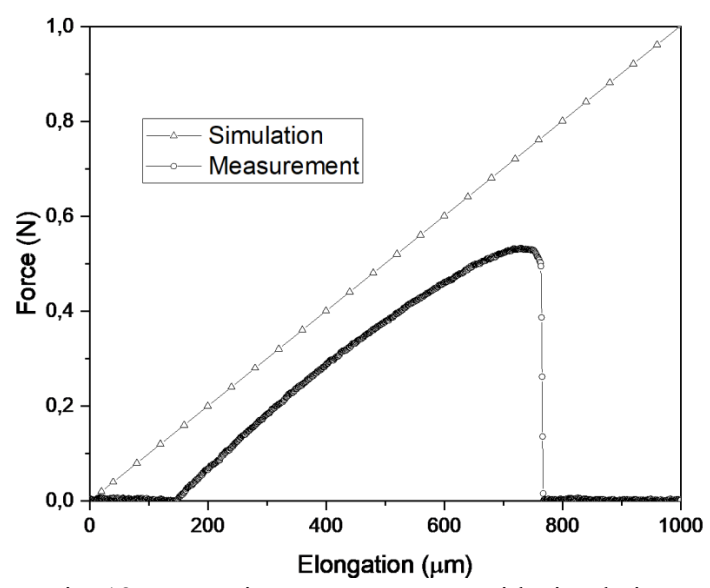

Fig. 12 Comparison measurement with simulation

Delamination of the metal has been studied based on CZM (Cohesive Zone Model) defining as parameters such as maximum contact normal stress and critical fracture energy $[10,11]$. Metal stress is chosen as input parameter together with CZM interface parameters. Fig. 13 shows deformed metal film due to delamination from parylene substrate and the deformed profile along with a side of the metal when metal film has residual stress of $230 \mathrm{MPa}$. Therefore, interface between metal film and parylene substrate should be carefully cared to achieve good adhesion.
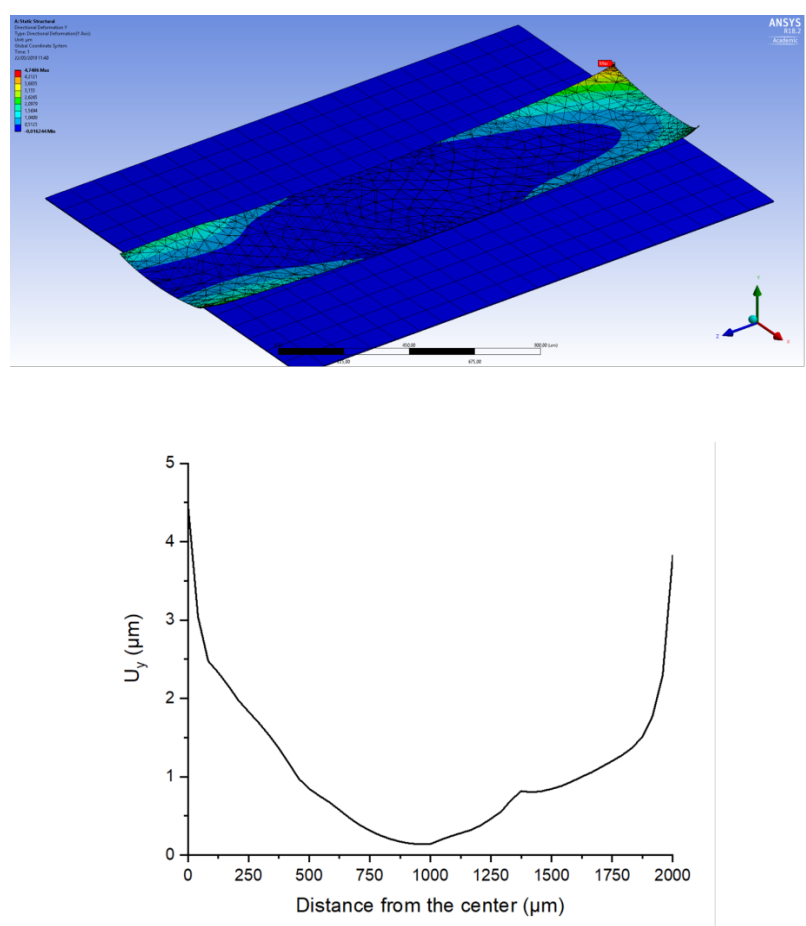

Fig. 13 Delamination of metal film on parylene substrate

\section{CONCLUSION}

Mechanical characteristics of parylen-metal-parylene system for implantable neural probe has been evaluated by using tensile strength measurement. Mean Young's modulus is 2.67 GPa similar value to typical parylene material, while strain at break is estimated $1.7 \%$ which much lower than the expected value. Thus, more studies are needed for such lower elongation at break. Metal delamination on parylene substrate has been found and adhesion force of the metal has been studied based on FEM modeling and simulation. Residual stress of the metal could be a reason of the metal delamination even if parylene surface has been modified to enhance interface energy.

\section{REFERENCES}

[1] K. Najafi, J. Ji, and K. D. Wise, "Scaling limitation on silicon multichannel recoding probe", IEEE Transactions on Biomedcal Engineering, 37, pp. 1-11, 1990.

[2] P. K. Campell, et al, "A silicon-based, three-dimensional neural interface: manufacturing processes for an intracortical electrode array", IEEE Transations on Biomedical Engineering, 38, pp. 758-768, 1991.

[3] S. Takeuchi, et al, "3D flexible multichannel neural probe array", Journal of Micromechanics and Microengineering, 14, pp. 104-107, 2004.

[4] O. Kim, et al, "Novel Neural Interface Electrode Array for the Peripheral Nerve", International Conference on Rehabilitation Robotics (ICORR), 2017

[5] C. D. Lee, and E. Meng, "Mechanical properties of thin-film Parylenemetal-Parylen devices", Frontiers in Mechanical Engineering, 2015.

[6] Y. Xie, et al, "Improving adhesion strength between layers of an implantable parylene-C electrode", Sensors and Actuators A: Physical, 260, pp.117-123, 2017.

[7] N. Oliva, et al, "On the use of Parylene C polymer as substrate for periphral nerve electrode", Scientific Reports, 2018.

[8] J. H. Chang, et al, "Adhesion-enhancing surface treatments for parylene deposition", International Solid-State Sensors, Actuators and Microsystems Conference (Transducers 2011), 2011.

[9] Parylene properties chart for parylene N, C, D www.parylene.com/pdfs/PTC-Parylene_Properties_Chart.pdf

[10] Seonho Seok, "A Theoretical Study on Post-it-like Debonding Process for BCB Cap Transfer Packaging Based on FEM Simulation" IEEE Transaction on Components, Packaging, Manufacturing Technology (CPMT), Oct. 2015, pp.1417-1422.

[11] Seonho Seok, "Fabrication and Modeling of Nitride Thin Film Encapsulation Based on Anti-Adhesion-Assisted Transfer Technique and Nitride/BCB Bilayer Wrinkling", IEEE Transaction on Components, Packaging, Manufacturing Technology (CPMT), 2016, Vol.6, No.9, Sept 2016, pp.1301-1307. 